\title{
Médiévales
}

Langues, Textes, Histoire

68 | printemps 2015

Langues d'Angleterre

\section{Monnaie, valeur et citoyenneté chez Olivi et Eiximenis. « Moralisation de l'économie » ou « économie politique » médiévale?}

Money, value and citizenship in Olivi and Eiximenis. A "moralization of economy" or a medieval "political economy"?

\section{Clément Lenoble}

\section{OpenEdition}

Journals

Édition électronique

URL : https://journals.openedition.org/medievales/7500

DOI : $10.4000 /$ medievales. 7500

ISSN : $1777-5892$

\section{Éditeur}

Presses universitaires de Vincennes

\section{Édition imprimée}

Date de publication : 15 juin 2015

Pagination : 161-180

ISBN : 978-2-84292-430-0

ISSN : 0751-2708

\section{Référence électronique}

Clément Lenoble, «Monnaie, valeur et citoyenneté chez Olivi et Eiximenis. « Moralisation de l'économie » ou «économie politique » médiévale ? », Médiévales [En ligne], 68 | printemps 2015, mis en ligne le 15 juin 2017, consulté le 23 avril 2022. URL : http://journals.openedition.org/medievales/7500 ; DOI : https://doi.org/10.4000/medievales.7500 



\title{
Clément Lenoble
}

\section{Monnaie, valeur et citoyenneté chez Olivi et Eiximenis}

\author{
"Moralisation de l'économie" \\ ou «économie politique» médiévale?
}

Les récentes éditions et traductions (l'une en français, l'autre en italien) de deux traités écrits respectivement à la fin du XIII ${ }^{e}$ et du XIV ${ }^{e}$ siècle par deux frères mineurs alimentent les débats autour de l'histoire de l'argent et de la valeur, et, plus généralement, de l'économie médiévale ainsi que des liens entre ce que nous, Occidentaux, appelons de nos jours économie, religion et gouvernement à la fin du Moyen Âge: Sylvain Piron a proposé en 2012 une nouvelle édition critique, à partir de nouveaux manuscrits, du célèbre Traité des contrats de Pierre de Jean Olivi, et en 2013 Paolo Evangelisti a traduit et longuement commenté les chapitres 139-152, 193197 du Douzième Livre du chrétien écrit par le catalan Francesc Eiximenis ${ }^{1}$. Dans leurs introductions respectives, les auteurs répondent tous deux, mais sur des modes différents, à certaines des critiques qui sont parfois faites aux études médiévales dites d'histoire de la «pensée économique» et au statut que celles-ci accordent à ces textes ${ }^{2}$.

Ces deux éditions-traductions participent d'une certaine actualité de la recherche sur les rapports entre économie, religion et politique à la fin du Moyen Âge et au début de l'époque moderne ${ }^{3}$. Elles sont en outre suivies

1. Pierre de Jean Olivi, Tractatus de contractibus, éd., trad. et comm. S. Piron, Traité des contrats, Paris, 2012; FrANCESC EIXIMENIs, Dotzè del Crestià (chap. 139-152 et 193-197), éd., trad. et comm. P. Evangelisti, Il Dodicesimo libro del Cristiano. Capp. 139-152 e 193197. Lo statuto della moneta negli scritti di un frate Minore del secolo XIV, Trieste, 2013.

2. Pour les critiques les plus récentes, B. Clavero, La Grâce du don. Anthropologie catholique de l'économie moderne, Paris, 1996 (1 $\left.1^{\mathrm{re}} \mathrm{e} d .1991\right)$; A. GuERrEAu, «Avant le marché, les marchés : en Europe, $\mathrm{XIII}^{\mathrm{e}}-\mathrm{XVIII}{ }^{\mathrm{e}}$ siècle», Annales. Histoire, Sciences Sociales, 56/6 (2001), p. 1129-1175 ; pour les plus récents, J. LE GoFf, Le Moyen Âge et l'argent. Essai d'anthropologie historique, Paris, 2010; et aussi L. Fontaine, Le Marché. Histoire et usages d'une conquête sociale, Paris, 2014.

3. J. KAYE, A History of Balance, 1250-1375: The Emergence of a New Model of Equilibrium and Its Impact on Thought, Cambridge, 2014; M. CARBONI, Il credito disciplinato. 
de près, en France, par la traduction d'un ouvrage de Giacomo Todeschini, sous le titre $A u$ pays des sans-nom, qui mobilise ces deux traités parmi de nombreuses autres sources ${ }^{4}$. On tentera donc d'exposer les enjeux et l'actualité historiographiques dans lesquels elles s'insèrent. Ceux-ci sont généralement posés dans les termes des débats autour des origines du capitalisme et de l'existence ou non d'une pensée économique médiévale à laquelle on oppose souvent le concept de morale économique chrétienne, jugé plus adapté au Moyen Âge. Ces deux ouvrages s'inscrivent au contraire dans une veine historiographique permettant de sortir de l'alternative entre, d'une part, une interprétation selon laquelle la scolastique aurait été le berceau de la pensée économique moderne et, d'autre part, une lecture, dont Jacques Le Goff fut le principal représentant français, niant l'existence de toute pensée de type économique au Moyen Âge et considérant que l'Église avait freiné puis encadré et moralisé l'essor du capitalisme marchand ${ }^{5}$. Ces études identifièrent souvent chez tel ou tel auteur le précurseur de l'économie de marché ou l'artisan de la synthèse entre celle-ci et les valeurs chrétiennes. Si Paolo Evangelisti et Sylvain Piron consacrent leur ouvrage chacun à un texte d'auteur, ils les replacent dans des contextes intellectuels, politiques et sociaux - sans doute plus vastes et précis, grâce aux progrès de la science, que ceux envisagés notamment par Jacques Le Goff - permettant de penser leur spécificité et leur place dans l'histoire des discours sur la richesse, la monnaie, la valeur et les échanges matériels.

Le traité de Pierre de Jean Olivi écrit à la fin du XIII ${ }^{\mathrm{e}}$ siècle (12931295) est, comme le rappelle Sylvain Piron, un manuel de confesseur, une œuvre de théologie morale consacrée aux différents types de contrats

Il monte di pietà di Bologna in età barocca, Bologne, 2014. Signalons aussi E. BAIN, Église, richesse et pauvreté dans l'Occident médiéval. L'exégèse des Évangiles aux XII'-XIII' siècles, Turnhout, 2014, paru trop récemment pour être pris en considération ici.

4. G. TodeschinI, Visibilmente crudeli. Malviventi, persone sospette e gente qualunque dal Medioevo all'Età moderna, Bologne, 2007 (Au pays des sans-nom. Gens de mauvaise vie, personnes suspectes ou ordinaires du Moyen Âge à l'époque moderne, Verdier, 2015).

5. Cf.,parmi d'autres, d'un côté, F.X. von FunK, «Über die ökonomische Anschauungen der mittelalterlichen Theologie», Zeitschrift für die gesamte Staatswissenschaft, 25 (1869), p. 125-175 ; E. SCHREIBER, Die volkswirtshaftliche Anschauungen der Scholastik seit Thomas von Aquin, Iéna, 1913; J. A. Schumpeter, Histoire de l'analyse économique. I. L'âge des

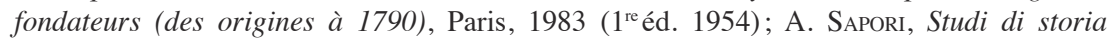
economica, I, Florence, 1955; R. DE Roover, La Pensée économique des Scolastiques, Montréal/Paris, 1971 ; de l'autre, C. Jourdain, Les Commencements de l'économie politique dans les écoles du Moyen Âge, Paris, 1874; E. Troeltsch, Die Soziallehren der christlichen Kirchen und Gruppen, Tübingen, 1911; A. FANFANI, Le origini dello spirito capitalistico in Italia, Milan, 1933; J. Le Goff, Marchands et banquiers du Moyen Âge, Paris, 2001 (1956); ID., La Bourse et la Vie. Économie et religion au Moyen Âge, Paris, 1986; ID., Le Moyen Âge et l'argent...; I ID., Préface de B. Clavero, La Grâce du don..., p. IX-XVII ; G. Barbieri, Fonti per la storia delle dottrine economiche dall'antichità alla prima Scolastica, Milan, 1958; J. Gilchrist, The Church and Economic Activity in the Middle Ages, New York, 1969. 
d'achats-ventes, à l'usure et à la restitution des profits mal acquis. Le frère du couvent de Narbonne règle les problèmes que le maniement de l'argent risque de poser pour le salut de l'âme et les équilibres sociaux ${ }^{6}$. Il déclare non peccamineuses mais aussi licites et acceptables au plan juridique un grand nombre de pratiques et de formes d'échanges contractuels. Ce faisant, il élabore un discours normatif, en particulier sur le crédit et les contrats, qui décrit la place centrale des marchands dans l'organisation de la cité ainsi que l'importance des activités économiques pour le «Bien commun» des citoyens. SylvainPiron souligne l'inventivité d'Olivi et la nouveauté d'une œuvre qui propose «une synthèse entre philosophie morale et principes juridiques » afin de «penser l'organisation d'ensemble d'un secteur particulier des relations sociales ${ }^{7} »$. Si Joseph Schumpeter a cru trouver là une anticipation des théories modernes qui lisent le monde comme un ensemble d'individus séparés cherchant chacun à maximiser leur profit par des stratégies et des calculs rationnels ${ }^{8}$, la pensée scolastique repose en réalité sur des postulats anthropologiques différents en fondant l'échange sur un principe éthique de justice et d'orientation vers le «Bien commun» de la collectivité ${ }^{9}$.

Un siècle plus tard, entre 1385 et 1391 , Francesc Eiximenis déclare que l'objectif du Dotzè libro del Crestià, où il aborde notamment les questions monétaires, est de faire œuvre de pédagogie civile et politique en direction du roi d'Aragon, commanditaire de l'œuvre, de ses successeurs et des hommes qui gouvernent les cités du royaume. Les réflexions sur la monnaie sont insérées dans un discours sur l'exercice du gouvernement où l'auteur reprend la matière d'un autre de ses textes écrit en 1383, le Regiment de la cosa publica. La monnaie est considérée comme le second élément constitutif de la civitas, après les nécessités alimentaires, avant la liberté, la paix, la sagesse, la science, la justice. Le frère catalan aborde les modalités du contrôle de la valeur, de la stabilité et de la circulation monétaire, les liens entre la majesté du prince et celle de la monnaie instituée pour garantir la justice et les échanges à l'intérieur de la cité. Le bien commun et la justice dérivent de la stabilité de la monnaie et sont le résultat du bon gouvernement du prince, des administrateurs du royaume et des cités.

6. S. PIRON, «Marchands et confesseurs. Le Traité des contrats d'Olivi dans son contexte (Narbonne, fin XIII ${ }^{\mathrm{e}}$-début XIV ${ }^{\mathrm{e}}$ siècle)», dans L'Argent au Moyen Âge. XVIII ${ }^{e}$ congrès de la SHMESP (Clermont-Ferrand, 30 mai-1 ${ }^{\text {er }}$ juin 1997), Paris, 1998, p. 289-308; ID., Pierre de Jean Olivi..., p. 39-49.

7. Ibid.,p. 21.

8. J. A. SChUmpeter, Histoire de l'analyse économique..., p. 144.

9. S. Piron, Pierre de Jean Olivi..., p. 25-26 et 43-49. 


\section{L'historiographie, entre doctrine sociale chrétienne et naissance du capitalisme}

La nature de ces discours et leur intérêt pour l'histoire économique ou pour l'histoire de la pensée économique font débat. En 1980, Giacomo Todeschini publiait une première édition du traité d'Olivi qu'il présentait comme «un traité d'économie politique franciscaine». Sept ans plus tard, Ovidio Capitani intitulait un recueil d'articles signés par différents auteurs «Une économie politique au Moyen Âge ${ }^{10}$ ». Ce choix provocateur du concept d' «économie politique» s'opposait à l'idée de «moralisation de l'économie » par l'Église médiévale, mais aussi à celle d'une contradiction entre morale chrétienne et réalités économiques, répandue dans l'historiographie depuis le XIX ${ }^{\mathrm{e}}$ siècle et jusqu'à Jacques Le Goff ${ }^{11}$. Depuis la fin des années 1970, une analyse globale des discours médiévaux sur la richesse a renouvelé notre vision des rapports entre économie, religion et gouvernement de la fin de l'Antiquité à l'époque moderne en dépassant les cloisonnements que la tradition avait instaurés entre histoire religieuse, histoire politique et histoire économique ${ }^{12}$. Elle reçut parfois des accusations d'anachronisme peu justifiées qui gauchissaient ses conclusions en s'arrêtant même parfois simplement à la terminologie des titres des publications ${ }^{13}$. Pourtant, elle

10. G. TodeschinI, Un trattato di economia politica francescana: il «De emptionibus et venditionibus, de usuris, de restitutionibus» di Pietro di Giovanni Olivi, Rome, 1980; O. Capitani éd., Una economia politica nel Medioevo, Bologne, 1987.

11. $C f$.n. 5, cela dans un sens qui n'a rien à voir avec l'économie morale d'E. P. Thompson, «The Moral Economy of the English Crowd in the Eighteenth Century», Past and Present, 50 (1971), p. 76-136.

12. G. Todeschini, «Oeconomica Franciscana. Proposte di una nuova lettura delle fonti dell'etica economica medievale», Rivista di storia e letteratura religiosa, 12 (1976), p. 15-77; ID., «Oeconomica Franciscana II. Pietro di Giovanni Olivi come fonte per la storia dell'etica economica medievale», Rivista di storia e letteratura religiosa, 13 (1977), p. 461-494; ID., Il prezzo della salvezza. Lessici medievali del pensiero economico, Rome, 1994; ID., I mercanti e il tempio. La società cristiana e il circolo virtuoso della ricchezza fra Medioevo ed Età moderna, Bologne, 2002; ID., Ricchezza francescana. Dalla povertà volontaria alla società di mercato, Bologne, 2004; ID., Visibilmente crudeli...; ID., Come Giuda. La gente comune e i giochi dell'economia all'inizio dell'epoca moderna, Bologne, 2011; G. Ceccarelli, Il gioco e il peccato. Economia e rischio nel Tardo Medioevo, Bologne, 2003; V. Toneatto, P. Černic, S. Paulitti, Economia monastica. Dalla disciplina del desiderio all'amministrazione razionale, Spolète, 2004; P. Evangelisti, I Francescani e la costruzione di uno Stato. Linguaggi politici, valori identitari, progetti di governo in area catalano-aragonese, Padoue, 2006; V. Toneatto, Les Banquiers du Seigneur. Moines et évêques face à la richesse (IV -début IX siècle), Rennes, 2012; pour la période moderne, J.-Y. GRenier, L'Économie d'Ancien Régime. Un monde de l'échange et de l'incertitude, Paris, 1996.

13. C'est particulièrement visible dans les critiques de B. Clavero, La Grâce du don, p. 20; J. Le Goff, Le Moyen Âge..., p. 121-122, 201-202, 207. Pour une lecture critique du livre de B. Clavero, $c f$. S. Piron, «Le devoir de gratitude. Émergence et vogue de la notion d'antidora au XIII ${ }^{\mathrm{e}}$ siècle», dans D. Quaglioni, G. TodeschinI, G. M. Varanin éd., Credito e 
s'opposait en réalité à l'historiographie évolutionniste du XIX et des trois premiers quarts du $\mathrm{xx}^{\mathrm{e}}$ siècle, dont les principaux objectifs avaient effectivement été de retracer soit les origines du capitalisme et de la pensée économique moderne soit celles de la doctrine sociale de l'Église, autrement dit d'un marché moralisé ou encadré et limité par les valeurs chrétiennes ${ }^{14}$.

Une autre «critique», reprise par Jacques Le Goff, fut de considérer que ces travaux ne se fondaient que sur un seul traité qui aurait été «bizarre», mineur, anecdotique et sans postérité, celui de Pierre de Jean Olivi sur les contrats et l'usure, ou du moins de ne pas faire preuve d'exhaustivité ${ }^{15}$. L'analyse des discours inaugurée par Giacomo Todeschini à travers des «chaînes lexicales» utilisées sur la très longue durée propose en réalité, sans nécessité d'exhaustivité (ce n'est pas une démarche proprement philologique ni une étude de filiation de doctrines), une vision panoramique qui n'est plus centrée sur un ou quelques auteurs ou sur un seul type de textes (le droit canonique, l'hagiographie, les sermons, l'exégèse.... ${ }^{16}$. Cette méthode a permis de retrouver, en les réinsérant dans les contextes institutionnels, politiques et sociologiques dans lesquels ils furent produits, les sens évolutifs et complexes, sur la très longue durée, de mots ou d'expressions polysémiques qui reliaient étroitement et de façon coessentielle aux dynamiques du salut et aux desseins divins les pratiques économiques terrestres, c'est-à-dire toute la sphère des actions quotidiennes, de la vie matérielle et des activités marchandes à plus grande échelle.

C'est pourquoi on lira avec curiosité les pages que consacre Laurence Fontaine à ces travaux ${ }^{17}$. Selon elle, ces historiens se contenteraient de «voir comment les abbés gèrent les biens terrestres dont ils ont la charge tout en favorisant le salut de l'âme de chacun, c'est-à-dire comment ils accordent leur mission spirituelle avec les besoins matériels des couvents et des hommes d'Église ${ }^{18}{ }$, ce qui n'est pas seulement une réduction à l'absurde mais une lecture erronée, et sans doute très rapide et partielle, des

usura fra ideologia, diritto e amministrazione. Linguaggi a confronto (sec. XII-XVI), Rome, 2005, p. 73-101.

14. Thèse défendue notamment par J. Le Goff. $C f$. les analyses historiographiques de G. Todeschini, Il prezzo..., p. 39-101 ; V. Toneatto, Les Banquiers..., p. 35-55 ; EAD., «La richesse des Franciscains. Autour du débat sur les rapports entre économie et religion au Moyen Âge», Médiévales, 60 (2011), p. 187-202.

15. J. Kirschner, K. Lo Prete, «Peter John Olivi's Treatises on Contracts of Sale, Usury and Restitution: Minorite Economics or Minor Works?», Quaderni Fiorentini, 13 (1984), p. 233-286; J. Le Goff, Le Moyen Âge et l'argent..., p. 122, 124.

16. ID., La Bourse ..., utilisait au contraire quasi exclusivement les exempla sur l'usure.

17. L. Fontaine, Le Marché..., p. 15-31 ( $c f$. la recension critique d'A. Skornicki, «Le marché, entre domination et émancipation», La Vie des idées, 16 janvier 2015, en ligne: http://www.laviedesidees.fr/Le-marche-entre-domination-et-emancipation.html).

18. Ibid., p. 21. Cette interprétation semble tirée en partie de L. FelLeR, «Sur la formation des prix dans l'économie du haut Moyen Âge», Annales. Histoire, Sciences Sociales, 66/3 (2011), p. 627-661 (p. 645) qui résume cependant un certain nombre d'autres conclusions des 
conclusions de tous ces travaux. Cette interprétation a néanmoins l'intérêt de révéler la difficulté, largement répandue dans l'historiographie, de penser autrement que dans les termes qui les définissent depuis le $\mathrm{XIX}^{\mathrm{e}}$ siècle, les rapports entre ce que nous considérons depuis comme les sphères autonomes, voire antinomiques, de la religion et de l'économie. Autrement dit, cette lecture restrictive montre bien la difficulté de se défaire d'une conception du christianisme médiéval vu comme un frein, un ensemble de principes spirituels et moraux en contradiction avec le monde réel des pratiques, comme si les moines et les évêques, coupés du reste du monde social, avaient dû composer avec les réalités de l'économie préexistant à l'état quasi naturel au sein de la société laïque ${ }^{19}$.

En voulant corriger par un effort de contextualisation les analyses des médiévistes qui se sont penchés sur ces problèmes, l'auteur croit déceler une «économie politique »: l'Église de la fin de l'Antiquité et du haut Moyen Âge brimait le marché et condamnait sévèrement toute forme d'enrichissement et d'activité économique car elle cherchait à s'affirmer vis-à-vis des deux autres ordres qui composaient, selon elle, la société au moment où Benoît de Nursie écrivait sa règle monastique: «la noblesse et le Tiers-État ${ }^{20}$ ». Faut-il comprendre que la «théorie» des trois ordres, voire celle des états, existait et correspondait réellement à l'organisation sociale de l'Occident à la fin de l'Antiquité? Laurence Fontaine ne s'arrête pas là. Dès le $\mathrm{VI}^{\mathrm{e}}$ siècle, les moines auraient condamné les laïcs (rappelons que les moines n'étaient pas des clercs), ceux-ci réduits aux marchands qui s'enrichissaient grâce au marché et que l'Église aurait cherché peu à peu à «encadrer». En condamnant l'avarice, affirme-t-elle, ils ne s'opposaient qu' aux plus riches (mais de qui parlaient-ils lorsqu'ils fustigeaient l'avarice des pauvres? on se le demande). Et puis un beau jour, neuf siècles plus tard, Bernardin de Sienne aurait réalisé fort heureusement, parce que c'est l'évidence même, que le microcrédit était la meilleure solution pour soulager la misère. C'est ainsi qu'auraient été inventés ces instruments d'ascension sociale que sont les monts-de-piété. Et l'Europe entrerait bientôt dans une économie de marché autorégulée ${ }^{21}$. Et voici revenir - après trois citations isolées de leur contexte, quelques approximations chronologiques et une vague idée de l'histoire du Moyen Âge - la vieille thèse, autrefois chère à une certaine érudition positiviste et évolutionniste, cette fois-ci sans

analyses dont il est question ici, notamment à propos de la circulation de la richesse et de la portée sociale et politique de ces discours.

19. On trouve la même idée chez J. Le Goff, La Bourse...; ID., Le Moyen Âge et l'argent..., p. 233 par exemple.

20. L. Fontaine, Le Marché..., p. 22.

21. Contra G. Todeschini, «Credibilità, fiducia, ricchezza: il credito caritativo come forma della modernizzazione economica europea », dans P. Avallone éd., Prestare ai poveri. Il credito e i Monti di Pietà in area Mediterranea (secoliXV-XIX), Naples, 2007, p. 17-30. 
érudition, du capitalisme d'abord rejeté et finalement promu et moralisé par l'Église. On trouve au fond à peu près les mêmes méthodes pour arriver plus ou moins aux mêmes conclusions chez Rodney Stark et chez les tenants de l' «Economy of religion » ${ }^{22}$. Car Laurence Fontaine, manifestement trop occupée à démontrer, comme eux, les vertus libératrices du marché, ignore aussi que la transformation au XVI ${ }^{\mathrm{e}}$ siècle des monts-de-piété en banques de dépôts pour les oligarchies marchandes permit à celles-ci de mettre la main sur le petit crédit à la consommation, comme le montre bien en revanche le livre que vient de publier Mauro Carboni sur Bologne ${ }^{23}$.

Ces «débats» n'ont pas vraiment lieu d'être, à condition que l'on se donne la peine de lire les travaux incriminés et de regarder de près les sources sur lesquelles ils fondent leur méthode d'histoire des discours et du vocabulaire, ce que les éditions et les traductions de PaoloEvangelisti et de Sylvain Piron permettent de faire plus facilement qu'auparavant, au moins pour deux traités majeurs de la fin du Moyen Âge.

\section{Des Pères aux théologiens franciscains: I'historiographie à l'épreuve des textes}

Les fondements lexicaux et conceptuels de nombre des textes utilisés par les deux théologiens franciscains sont anciens. Dès la fin de l'Antiquité, les Pères de l'Église considérèrent que l'argent n'était en soi ni bon ni mauvais. À partir de leur exégèse de la parabole des talents, ils déterminèrent qu'il pouvait être bénéfique pour la communauté et l'âme de celui qui l'utilisait. Et l'intérêt, bien évidemment, n'est pas de constater que les Pères cherchaient à concilier le salut de l'âme avec les besoins matériels de l'Église, qu'ils connaissaient le sens du mot profit ou qu'ils inventèrent le marché, mais bien plutôt de noter la portée politique de leurs discours sur la richesse. Ce n'était pas l'argent qui devait être repoussé, mais les comportements avaricieux envers celui-ci, autrement dit le désir compulsif de richesse, son accumulation néfaste pour la communauté. L'importance du bon usage de l'argent et des richesses en général, présenté comme une absence d'accumulation propriétaire et comme une mise en circulation par un lexique fondé sur des catégories théologiques comme

22. On s'étonne de ne pas les trouver en bibliographie tant les positions sont proches: R. B. Ekelund Jr, R. F. Hébert, R. D. Tollison, The Marketplace of Christianity, Cambridge (Mass.) et Londres, 2006; R. STARK, The Victory of Reason: How Christianity Led to Freedom, Capitalism and Western Success, Londres, 2005 (trad. fr. Le Triomphe de la raison. Pourquoi la réussite du modèle occidental est le fruit du christianisme, Paris, 2007). Cf. le compte rendu critique de B. MichON, «Le triomphe de la raison. Pourquoi la réussite du modèle occidental est le fruit du christianisme», Archives de sciences sociales des religions, 140 (2007), en ligne: http://assr.revues.org/document11973.html.

23. M. CARBoni, Il credito disciplinato... 
la dispensatio et la caritas $^{24}$, servit à cette époque à la définition de la communauté des fidèles puisque les mauvais comportements dans ce domaine, propres aux avari, étaient attribués aussi aux infidèles ou aux hérétiques, identifiant ainsi tous ceux qu'il fallait exclure de la communion, en tant que non-chrétiens ou mauvais chrétiens, comme de mauvais usagers de la richesse terrestre et céleste. Les discours sur l'usage de la monnaie et l'échange des choses matérielles contaminèrent ceux qui affirmaient les prérogatives épiscopales en matière de gouvernement des communautés et d'administration temporelle. Ils furent intégrés aux règles monastiques pour définir le gouvernement de l'abbé et des officiers du monastère, l'obéissance des moines envers eux et la supériorité monastique en matière de gestion des biens. Technique administrative, l'usage des res sacrae était défini comme l'un des éléments de l'ascèse. Si elles étaient déviantes, ses modalités étaient objet de correction fraternelle et de pénitence au sein des monastères. Les Pères élaborèrent dans ce domaine un langage d'autorité à vocation normative qui contribuait à définir la communauté chrétienne comme un corps à la fois religieux, politique et économique, au sein duquel moines et évêques faisaient figure de guides, d'experts et de modèles pour tous les autres ${ }^{25}$.

Sur ce socle lexical, réemployé pendant tout le haut Moyen Âge et réélaboré pendant les débats autour de la simonie et de l'usure durant le Moyen Âge central dans le contexte des polémiques ouvertes par les volontés de réforme de l'Église, la question de l'usage de l'argent prit place dans les transformations de la pénitence à travers la pratique de la confession ${ }^{26}$. Et

24. J. LeGoff a souvent insisté sur le rôle de la caritas comme moteur des échanges à la fin du Moyen Âge mais pas sur la dispensatio, action et qualité pourtant fondamentale de ceux qui participent aux échanges. Sur la caritas, souvent invoquée et rarement définie, voir G. TodeschinI, I mercanti..., p. 326 sq. ; V. Toneatto, Les Banquiers..., p. 139-155. Sur la dispensatio, ibid., p. 290 sq.

25. V. Toneatto, Les Banquiers... pour l'ensemble de ce paragraphe. Signalons que cet ouvrage est tiré d'une thèse de doctorat soutenue en 2009 et s'appuie sur certaines des conclusions publiées en 2004 par l'auteur dans EAD., «I linguaggi della ricchezza nella testualità omiletica e monastica dal III al IV secolo», dans V. TONEATTO et al., Economia monastica..., p. 1-88, et en 2010 dans EAD., «Diligenter et fideliter. Linguaggi monastici della razionalità economica tra Oriente e Occidente (IV-VIsec.)», dans A. De VInCENTIIs éd., Il moderno nel Medioevo. Seminari dell'Istituto Storico Italiano per il Medioevo 2005-2006, Rome, 2010, p. 175-199.

26. Pour l'histoire des discours sur l'argent dans la très longue durée, $c f$. G. ToDESCHINI, Il prezzo..., p. 163-211 et ID., I mercanti... Sur les transformations de la pénitence et de la confession, $c f$. en particulier P. PRODI éd., Disciplina dell'anima, disciplina del corpo e disciplina della società tra medioevo ed età moderna, Bologne, 1994 ; ID.,Una storia della giustizia. Dal pluralismo dei fori al moderno dualismo tra coscienza e diritto, Bologne, 2000 ; A. ProsPeri, Tribunali della coscienza. Inquisitori, confessori, missionari, Turin, 1996; J. Chiffoleau, La Chiesa, il segreto e l'obbedienza. La costruzione del soggetto politico nel medioevo, Bologne, 2010; A. Fossier, La Fabrique du droit. Casuistique, qualifications juridiques et pratiques administratives de la pénitencerie apostolique (début XIII-début 
ce n'est pas un hasard si le traité d'Olivi sur les contrats est un manuel de confesseur. L'intérêt des confesseurs et des théologiens pour ces problèmes se développa dans le contexte de l'émergence et de l'affirmation d'un groupe professionnel qui prenait de plus en plus d'importance, depuis le $\mathrm{XII}^{\mathrm{e}}$ siècle, dans le gouvernement des cités et des royaumes. Mais il faut aussi tenir compte des effets de l'entrée de ces pratiques dans le champ de la confession et de la pénitence. On n'a sans doute jamais suffisamment insisté sur le fait que l'interdiction de l'usure, la condamnation de l'usurier manifeste et l'infini raffinement casuistique de la réflexion des théologiens et des juristes sur les contrats et l'argent qu'elles engendrèrent à partir du $\mathrm{XII}^{\mathrm{e}}$ siècle, avaient peut-être moins eu pour effet et objectif de bloquer puis de libérer l'essor économique de l'Occident latin que d'étendre la juridiction des clercs et le modèle de l'administration sacrée des res ecclesiae sur une infinité de choix quotidiens et de pratiques professionnelles de l'ensemble des fidèles. Ce faisant, l'Église rendait vertueuses les techniques et les activités des grands marchands, alors que celles des gens ordinaires et de l'économie quotidienne étaient suspectées d'usure ${ }^{27}$.

Si l'on peut penser avec Jacques Le Goff qu'un usurier en purgatoire ne fit pas le capitalisme, en revanche, la migration de l'usurier de l'enfer au purgatoire n'avait pas pour fonction de limiter les excès des plus riches ${ }^{28}$. L'usurier n'est pas l'ancêtre du banquier. Nous savons aujourd'hui que les grands marchands et hommes d'affaires qui prêtaient de l'argent, parfois à des taux exorbitants, ne furent que très rarement accusés de pratiquer l'usure ${ }^{29}$. Cette qualification répondait à d'autres logiques, plus politiques

$X V^{e}$ siècle), thèse soutenue sous la direction de J. Chiffoleau, EHESS, 2012, à paraitre dans la Bibliothèque des Écoles françaises de Rome et d'Athènes sous le titre Le Droit bien tempéré. Casuistique et pratiques administratives de la Pénitencerie Apostolique (début XIIt -début $X V^{e}$ siècle). Sur les liens entre activité marchande et pénitence, S. PIRON, «Marchands...»; B. Molina, G. Scarcia éd., Ideologia del credito fra Tre e Quattrocento: dall'Astesano ad Angelo da Chivasso, Asti, 2001; O. Langholm, The Merchant in the Confessional: Trade and Price in the Pre-Reformation Penitential Handbooks, Leyde, 2002; D. Quaglioni, G. Todeschini, G.-M. Varanin éd., Credito e usura fra teologia, diritto e amministrazione. Linguaggi a confronto (sec. XII-XVI), Rome, 2005.

27. G. Todeschin, Visibilmente crudeli..., p. 105 sq. ; ID., Come Giuda..., p. 233 sq.

28. J. Le Goff, La Bourse..., p. 98.

29. $C f$. par exemple D. Kusman, «Jean de Mirabello dit van Haelen (ca 1280-1330). Haute-finance et Lombards en Brabant dans le premier tiers du XIV siècle», Revue belge de philologie et d'histoire, 77/4 (1999), p. 843-931 (p. 867-869); ID., Usuriers publics et banquiers du Prince. Le rôle économique des financiers piémontais dans les villes du duché de Brabant (XIII - -XIV siècle), Turnhout, 2013; R. BORDONE, «Tra credito e usura: il caso dei Lombardi e la loro collocazione nel panorama economico dell'Europa medievale», dans G. Boschiero, B. Molina éd., Politiche del credito. Investimento, consumo, solidarietà, Asti, 2004, p. 141-161; M. GiAnsANTE, L'usuraio onorato. Credito e potere a Bologna in età comunale, Bologne, 2008. 
et sociales, que celle du simple montant du profit usuraire ${ }^{30}$. Moralisation, donc, mais dans le sens où la réflexion des scolastiques faisait entrer ces pratiques nouvelles dans le champ de la théologie morale.

\section{Monnaie, citoyenneté et inégalités}

L'analyse de Paolo Evangelisti souligne cet aspect du discours d'Eiximenis sur l'argent et le statut de la monnaie qui se traduit par la nécessité de garantir les équilibres sociaux et la justice à l'intérieur de la cité. Mêmes remarques chez Sylvain Piron au sujet d'Olivi dont le traité était orienté vers la justice et l'équité entre les marchands, notamment à travers l'usage des contrats et les techniques du juste prix pour établir la valeur des choses. Mais cette équité n'était pas une conception égalitaire des rapports entre les citoyens sur le mode arithmétique: Olivi se fonde sur Bonaventure pour affirmer que le superflu varie selon la qualité et la condition des personnes, ce qui justifie l'échelle des stipendia entre le duc, le cavalier et la piétaille. Les offices les plus élevés exigent plus d'exercice, de compétences, de labeur et d'intelligence, ce dont peu d'hommes sont capables. Ces «personnes éminentes» doivent être maintenues avec faste et révérence dans leur supériorité pour l'utilité de la communauté politique ${ }^{31}$. Intérêt collectif et intérêt individuel de certains membres du groupe sont ainsi étroitement associés. Tout comme Olivi considère que le juste prix est celui défini par la communauté des marchands dans une certaine «latitude» selon les circonstances de l'échange, les rapports de justice entre les parties semblent relativement «géométriques» ou plastiques dans la mesure où la justice du contrat, que l'on interprète parfois comme une forme de justice sociale, placée au fondement de la communauté et de l'échange dépend, elle

30. G. Todeschini, Visibilmente crudeli..., p. 105 sq. ; G.-M. VARAnini, «Condanne inquisitoriali, usura e politica fra Duecento e Trecento. Appunti sul caso veronese», dans G. Pullino, P. Pecorari, G.-M. Varanini éd., Scritti di storia economica e sociale in onore di Giovanni Zalin, Caselle di Sommacampagna (Vérone), 2011, p. 381-392.

31. Bonaventura, De superfluo, éd. E. Lio, S. Bonaventura e la questione autografa "De superfluo» contenuta nel ms. di Assisi, Bibl. comun. 186 citata dal Concilio Vat. II. Testo con studio critico-letterario e dottrinale, Rome, 1966, p. 157-159: «Est enim superfluum naturae quod est ultra naturae necessitatem, tamen potest esse quod non sit superfluum quantum ad conditionem personae, quia infirmus vel nobilis »; Pierre de JeAn Olivi, Tractatus..., éd. S. Piron, p. 114-116: «Quarto observat communem gradum et ordinem officiorum et dignitatum eis annexarum, unde et maiora stipendia dantur duci quam militi, et militi quam scutifero vel pediticuius racio est triplex [...] quod ad altiora officia debite exequenda exigitur maior pericia et industria et amplior sollicitudo mentalis [...] et eciam quia multo et duiturno studio ac experiencia et labore, multisque periculis et expensis communiter adquiritur pericia et industria talis, et eciam quia rari et pauci sunt ad hoc idoney, et ideo in maiori precio reputantur. [...] Ad honorem et utilitatem civilis communitatis facit quod superiores reverencius et cumulacius in quadam sensibili superioritate et dignitate serventur [...]. » 
aussi, de plusieurs critères et d'une évaluation commune de ces critères qui sont à la fois religieux, sociaux et conjoncturels ${ }^{32}$.

Chez Eiximenis, ce même type de conception est intégré à un discours sur la monnaie qui est indissociable de celui sur le pouvoir du souverain, la nature des monarchies et des res publicae, et de celui sur les qualités nécessaires au statut de citoyen qui sont développés au sein du Douzième Livre. Tout candidat à la citoyenneté doit acquérir et montrer ostensiblement une disposition pour la res publica qui est associée étroitement aux capacités de gestion de la richesse monétaire. Au sein d'une communauté civique définie comme la communauté à l'intérieur de laquelle se réalisent les échanges contractuels (mariages, achat-ventes et prêts), la dignité de citoyen dépend de la capacité à participer à ces échanges et à en comprendre les règles ${ }^{33}$. Doivent donc être exclus de la citoyenneté tous ceux qui ne participent pas aux échanges, exercent des activités considérées comme non fructueuses ou inutiles pour la communauté, sont incapables d'adapter leur comportement économique aux objectifs définis par la communauté ${ }^{34}$. À partir de quoi, différents niveaux de citoyenneté sont établis en fonction des propriétés et de l'utilité de chaque profession. Le marchand expert des échanges contractuels assume une fonction de structuration civile essentielle et représente le modèle de la bonne citoyenneté. «Miroir» de la communauté, le citoyen enrichi participe nécessairement au bien être de tous. Les citoyens, par conséquent, ne sont pas égaux entre eux, ni en richesse ni en matière de citoyenneté. Selon le frère mineur catalan, l'égalité bloquerait les échanges et les contrats, donc la société entière: la propriété

32. Pierre de Jean Olivi, Tractatus..., éd. S. Piron, p. 96-103: «[...] communi saluti hominum post lapsum expedit quidem ut taxacio precii rerum venalium non sit punctualis, nec secundum absolutum valorem rerum, sed potius ex communi consensu utriusque partis, vendencium scilicet et emencium, libere pretaxetur [...] res non possunt licite vendi plus quam valeant, nec minus emi, pensato earum valore in respectu ad usum nostrum et ad probabile iudicium humane extimacionis mensurantis valorem rei infra limites latitudinis competentis. » Sur la plasticité du juste prix et ses conséquences, $c f$. J. W. BALDwin, The Medieval Theories of the Just Price. Romanists, Canonists and Theologians in the Twelth and Thirteenth Centuries, Philadelphie, 1959, p. 54-57; S. PIRON, «Vœu et contrat chez Pierre de Jean Olivi », Cahiers du Centre de recherches historiques, 16 (1996), p. 435-446; ID., «Perfection évangélique et moralité civile. Pierre de Jean Olivi et l'éthique économique franciscaine», dans B. Molina, G. Scarcia éd., Ideologia..., p. 103-143; J. KaYe, Economy and Nature in the Fourteenth Century: Money, Market Exchange, and the Emergence of

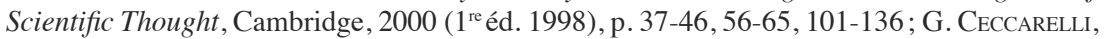
Il gioco..., p. $221 s q$. 90.

33. F. Eiximenis, Dotzè Llibre del Crestià, I. 1, éd. X. Renedo et al., Gérone, 2005, p. 89-

34. Ibid., p. 178, 186. Cf. P. Evangelisti, «Ad invicem participancium. Un modello di cittadinanza proposto da Francesc Eiximenis, frate francescano», dans C. LENOBLE, G. Todeschini éd., Cittadinanza e disuguaglianze economiche: le origini storiche di un problema europeo (XIII-XVI secolo), Mélanges de l'École française de Rome - Moyen Âge, 125/2 (2013), en ligne: http://mefrm.revue.org/1466. 
commune n'est pas la forme adéquate de la res publica au contraire de la propriété individuelle ${ }^{35}$.

Toutefois, la communauté ne s'enrichit que si chacun fait preuve de sobriété, de modération et d'autocontrôle dans la dépense en évitant le superflu dans l'alimentation et le vêtement ${ }^{36}$. La position d'Eiximenis rappelle les statuts des cités italiennes contre le luxe. Cet éloge de la modération, et la condamnation de l'excès qui l'accompagne, est moins une bataille contre les riches qu'une injonction à un usage technique, conscient et mesuré des ressources qui se fonde, dans la plus pure tradition franciscaine, sur une conception géométrique du superflu - nous venons de le voir avec Bonaventure et Olivi - selon laquelle la possession et l'usage des richesses dépendent du status de ceux qui savent et doivent les gérer ${ }^{37}$. Le principe de modération comme technique de gestion exclut les pauvres qui n'ont rien à gérer et tous ceux dont la situation peut être vue comme une incompétence économico-religieuse, un manque de fidélité et de fiabilité.

Ces théologiens franciscains ne furent pas les seuls à tenir ce type de discours. Joel Kaye a bien montré les rapports entre les conceptions monétaires médiévales comme instrument de mesure et de quantification de la valeur considérée comme relative et non comme absolue et le développement de nouveaux discours sur la nature qui privilégiaient une vision géométrique et mouvante du monde ${ }^{38}$. Tout récemment, il a mis en évidence la façon dont une conception organique du corps social supposait à la fin du Moyen Âge que les inégalités entre les membres ou les parties étaient nécessaires à l'harmonie naturelle de l'ensemble. De fait, les concepts de justice et d'équité n'impliquaient pas une égalité arithmétique entre les différents membres d'une communauté ${ }^{39}$.

Cette même analyse conduisit Eiximenis à formuler une véritable prophétie politique qui voue les monarchies à la disparition et célèbre l'avènement dans toute la chrétienté de res publicae entre les mains des marchands sur le mode des cités italiennes ${ }^{40}$. La monnaie est, dans cette vision de la société idéale, le bien de chacune de ces communautés. Sa souveraineté est donc supérieure à celle du prince qui ne peut intervenir sur sa valeur sans commettre un crime de lèse-majesté et qui ne peut qu'être le garant de cette valeur. Celle-ci est définie comme l'instrument de

35. F. Eiximenis, Dotzè Llibre del Crestià, I. 1, p. 192-193, 198-199, 210-212.

36. Ibid., cap. 197. Cf. P. Evangelisti, Il Dodicesimo libro..., p. 82-97; ID., «Ad invicem...».

37. ID., Il dodicesimo libro..., p. 97.

38. J. KAYE, Economy and Nature...

39. ID., A History of Balance...; ID., «Equalization in the Body and the Body Politic: From Galen to Marsilius of Padua», dans C. Lenoble, G. Todeschini éd., Cittadinanza..., en ligne: http://mefrm.revue.org/1252.

40. F. Eiximenis, Dotzè, I, 1, cap. 198-200, p. 428. 
construction de la res publica. La monnaie en est l'identité même. Comme chez le Franciscain Alexandre d'Alexandrie ou chez Nicole Oresme, la monnaie appartient à tous ceux qui la possèdent et l'utilisent selon les règles des échanges contractuels. Sa possession donne donc un droit à participer aux échanges et à délibérer sur sa valeur. La monnaie comporte en soi, pour tous ceux qui la possèdent et l'utilisent, une égalité de droit à la rémunération et à la décision. C'est donc à partir d'elle que peuvent se penser la communauté et le rapport des sujets avec le monarque. Alors que chez Thomas d'Aquin la citoyenneté est avant tout le fait de ceux qui participent au gouvernement de la res publica, la définition de la monnaie comme bien commun par Oresme et Eiximenis implique que sont citoyens tous ceux qui peuvent participer au gouvernement de la monnaie.

Le lien entre monnaie et citoyenneté est si étroit que le crédit public devient le moyen par excellence d'inclusion dans la communauté civique de nouveaux membres éventuellement issus de couches sociales moins favorisées qui peuvent en bénéficier: des citoyens ayant perdu momentanément leurs biens ou de jeunes cavaliers, de jeunes marchands sans capital suffisant, des artisans sans fonds, des paysans qui n'ont pas les moyens de cultiver la terre, à condition toutefois qu'ils soient réputés répondre, notamment à travers l'appartenance à des réseaux de relation, à un certain nombre de critères de fiabilité, de fidélité et de loyauté reconnus et partagés par la communauté et qu'ils soient jugés capables de participer à leur tour au profit commun. Autrement dit, contrairement à ce qu'ont affirmé avant lui Thomas d'Aquin et Nicole Oresme, la bonne citoyenneté selon Eiximenis n'est pas une qualité par excellence aristocratique, mais dérive davantage de mérites reconnus par la communauté des citoyens dans son propre intérêt qui peuvent s'acquérir par la participation aux échanges et l'usage de la monnaie.

En ce sens, la monnaie est considérée comme l'étalon, l'instrument de mesure de la citoyenneté. Celle-ci est à la fois multiple, en expansion potentielle, limitée, sélective et exclusive: «ces formes de financements non seulement excluent tout destinataire inactif mais servent explicitement à aider uniquement les professions qui "peuvent apporter un profit à la communauté" de sorte que "cela soit fait à l'avantage de Dieu et de la chose publique" ${ }^{41} »$. Les possibilités d'intégration ou d'ascension sociale par le crédit public sont donc limitées et «ne peuvent pas se réaliser non seulement pour une ample part des habitants de la civitas qui sont réputés infideles à la res publica, sans crédibilité et sans loyauté envers elle, mais

41. P. Evangelisti, «Ad invicem...», § 37: «Queste forme di finanziamento quindi non solo escludono qualsiasi destinatario inattivo ma valgono dichiaratamente ad aiutare solo le professioni che "possono portare profitto alla comunită" di modo che "ciò sia fatto $a$ vantaggio di Dio e a vantaggio della cosa pubblica" » (en italique, F. EIXIMENIS, Dotzé Llibre del Crestià, II. 1, éd. C. WitTlin et al., Gérone, 1986, p. 439-440). 
aussi pour le groupe des non fideles en raison de leur appartenance religieuse qui, comme les hérétiques, sont exclus de tout degré de citoyenneté ${ }^{42}$ ». La monnaie est donc autant un critère et un vecteur d'inclusion que d'exclusion, chez Eiximenis, dans la mesure où, comme Oresme, il considère le droit de la communauté des citoyens sur la monnaie comme co-essentiel à la citoyenneté ${ }^{43}$. Il fait ainsi de la monnaie une institution fondatrice de l'existence même de la communauté, en tant que conséquence d'une décision commune, élément de cohésion et objet de consensus, presque principe «constitutionnel», pour reprendre une expression de Paolo Evangelisti.

Cette lecture politique des fonctions et du statut de la monnaie fondatrice de la communauté civique, héritière des réflexions sur la société chrétienne comme une communauté de contractants dont Olivi fut bien l'un des artisans, est absente, par exemple, de la doctrine politique de Marsile de Padoue que les historiens de la pensée politique prennent pourtant généralement comme objet principal d'étude et de référence ${ }^{44}$. Reste à savoir si et dans quelle mesure ces idées, les concepts et les mots qui les façonnaient, étaient diffus et partagés, s'ils sortirent relativement des cercles intellectuels et ecclésiastiques, en l'occurrence franciscains, et s'ils eurent non pas peut-être une réelle hérédité, puisqu'il ne s'agit pas de tomber dans le piège des filiations de doctrines ou de «l'obsession embryogénique ${ }^{45}$ » pour parler comme Marc Bloch, mais du moins un certain écho.

\section{La longue durée des mots et les écarts avec la science économique}

Le premier travail publié par Giacomo Todeschini sur le traité des contrats d'Olivi en 1977 et l'édition qu'il proposa de ce texte quelques années plus tard montraient le substrat lexical complexe et très ancien, théologique et juridique, de ce traité, autant qu'ils pointaient les remplois ultérieurs de plusieurs concepts, catégories ou idées par les maîtres franciscains jusqu'à Bernardin de Sienne et Angelo da Chivasso. Trois ouvrages essentiels démontrèrent ensuite que certains éléments de la pensée d'Olivi en matière d'argent et d'échanges n'étaient pas du tout isolés ni uniques, non seulement au sein de l'ordre des frères mineurs, mais aussi dans l'Église médiévale

42. Ibid., $\S 43$ : «La mappa della cittadinanza prevede quindi alcune, limitate, integrazioni che tuttavia non possono realizzarsi non solo per una gamma molto ampia di abitatori della civitas che risultino infideles alla res publica, privi di credibilità e di lealtà verso di essa, ma anche per tutta quella schiera di non fideles per appartenenza religiosa che, insieme agli eretici, costituiscono un novero di esclusi da qualsiasi grado di cittadinanza. »

43. Cf. G. Todeschini, «Il denaro e l'esclusione sociale nel pensiero francescano », dans A. Cacciotti, M. Melli éd., I francescani e l'uso del denaro, Milan, 2011, p. 41-60, sur l'argent comme facteur d'exclusion chez les franciscains.

44. Sur Marsile, $c f$. les travaux récents de G. Briguglia, Marsilio da Padova, Rome, 2013 (trad. fr. Marsile de Padoue, Paris, 2014).

45. . B. Bоch, Apologie pour l'histoire ou Métier d'historien, Paris, 1952, p. 6. 
pendant les deux derniers siècles du Moyen Âge, tout en signalant l'infinie diversité et le raffinement technique et lexical des positions dans ce domaine ${ }^{46}$. Grand spécialiste de l'œuvre d'Olivi, Sylvain Piron réaffirme l'ampleur de la culture théologique du frère de Narbonne. Contrairement à ce qu'a pu affirmer Jacques Le Goff, ce traité n'a rien de «bizarre», mais correspond au contraire à «l'état normal d'une science» et «s'inscrit assurément dans le cadre des traditions intellectuelles du XIII ${ }^{\mathrm{e}}$ siècle $^{47} \gg$. La spécificité des positions d'Olivi sur la pauvreté volontaire n'en faisait pas forcément l'auteur d'un traité des contrats aux conceptions particulièrement exceptionnelles pour son époque, malgré toute son inventivité et malgré des différences notables entre certaines des positions d'Olivi et celles d'autres auteurs. Ainsi, l'un des principaux opposants aux idées d'Olivi et des «spirituels» au sujet de la règle des frères mineurs et de la pauvreté volontaire, le ministre général franciscain Guiral Ot, écrivit à son tour un traité des contrats dans lequel il reprenait nombre de solutions oliviennes ${ }^{48}$.

Paolo Evangelisti identifie quant à lui quatre grands filons de références citées, commentées et utilisées par Eiximenis. Le premier est juridique, civil et canonique, le second est constitué des commentaires et de l'analyse de l'œuvre d'Aristote, en particulier l'Éthique à Nicomaque, le troisième des traités sur la monnaie écrits au XIV siècle dans l'ère catalanoaragonaise, et le quatrième de celui de Nicole Oresme écrit quelques années plus tôt. Paolo Evangelisti ajoute, à ces quatre groupes de références explicitement commentées par le frère catalan, toute la réflexion théologique franciscaine sur la pauvreté volontaire et la richesse qui circulait dans les écoles des couvents, dont le traité sur les contrats d'Olivi qui était connu de ce côté des Pyrénées. Les maîtres franciscains produisirent en effet une importante littérature sur le statut et l'usage de l'argent, notamment dans le cadre de la querelle qui les opposa aux maîtres séculiers de l'Université de Paris pendant la seconde moitié du $\mathrm{XIII}^{\mathrm{e}}$ siècle $^{49}$. Paolo Evangelisti rappelle également la communauté d'idées et de lexique qui unissait les franciscains et les membres des gouvernements des cités et des royaumes, de la Sicile

46. G. Todeschin, Il prezzo...; ID., I mercanti...; ID., Ricchezza ..., passim. L'analyse de G. Todeschini est fondée sur plus de 120 auteurs.

47. S. Piron, Pierre de Jean Olivi. Traité..., p. 20.

48. G. Ceccarelli, S. Piron, «Gerald Odonis'Economics Treatise», Vivarium, 47 (2009), p. 164-204.

49. R. Lambertini, «Pecunia, possessio, proprietas alle origini di Minori e Predicatori: osservazioni sul filo della terminologia», dans L'economia dei conventi dei Frati Minori e Predicatori fino alla metà del Trecento, $31^{\circ}$ Convegno internazionale di studi, Assisi 9-11 ottobre 2003, Spolète, 2004, p. 5-42; ID., "“Pecunia adsidua permutatione quodammodo extinguitur". Spunti per una definizione del denaro nel dibattito su usura e povertà francescana», dans R. LAMBERTinI, L. Sileo éd., I beni di questo mondo. Teorie eticoeconomiche nel laboratorio dell'Europa medievale, Porto, 2010, p. 215-229; G. TodeschinI, «Il denaro e l'esclusione... », p. 41-60. 
à Valence à partir de la fin du XIII ${ }^{\mathrm{e}}$ siècle au moins, lorsqu'il s'agissait de décrire les relations économiques et politiques ${ }^{50}$. Le traité d'Eiximenis était exposé, enchaîné, dans la salle du conseil de la cité de Gérone au moins depuis le $\mathrm{xv}^{\mathrm{e}}$ siècle. À la même époque, les œuvres du frère mineur étaient présentes en grand nombre dans les bibliothèques des marchands, des notaires et des juristes de Barcelone. Au siècle suivant, le texte d'une délibération du conseil de cette même cité s'appuie explicitement sur le chapitre 115 du Dotzè del Crestià ${ }^{51}$.

Ce sont des preuves de l'influence et de l'usage de ce type de littérature. Si ce genre de preuves directes est relativement rare pour d'autres traités, d'autres moyens de diffusion sont attestés. Le fait que le traité d'Olivi était un manuel de confesseur dont les idées furent diffusées, par exemple, dans les couvents du nord-est de la Péninsule italienne jusqu'au début de l'époque moderne, comme le montre une enquête récente de Giovanni Ceccarelli, laisse supposer que de nombreux confesseurs aient pu avoir l'occasion d'en discuter avec les fidèles qu'ils entendaient en confession ou à qui ils pouvaient servir de directeur de conscience ${ }^{52}$. Ce langage saturait aussi les sermons des prédicateurs ${ }^{53}$. On sait bien par ailleurs que les marchands possédaient de nombreux livres de théologie dans leurs bibliothèques ${ }^{54}$. Ceux qui écrivirent sur leur activité et leur profession partageaient avec les théologiens les mêmes idées et le même vocabulaire ${ }^{55}$. Plutôt qu'une filiation de doctrines, ou une transmission des idées d'un auteur en particulier à un autre, il faut imaginer qu'un certain nombre de conceptions, de façons de qualifier les choses et les actions

50. P. Evangelisti, Il dodicesimo..., ad indicem; ID., I Francescani e la costruzione di uno Stato. Linguaggi politici, valori identitari, progetti di governo in area catalanoaragonese, Padoue, 2006.

51. ID., Il dodicesimo..., p. 14-15, où l'on trouvera en note la bibliographie correspondante et les sources citées.

52. G. Ceccarelli, «Concezioni economiche dell'Occidente cristiano alla fine del medioevo: fonti e materiali inediti», dans F. AMmANnATI éd., Religione e istituzioni religiose nell'economia europea. 1000-1800, Florence, 2012, p. 271-280; S. PIRON, « Marchands...»; O. LANGHOLM, The Merchant...

53. N. BÉRIOU, «L'esprit de lucre entre vice et vertu. Variations sur l'amour de l'argent dans la prédication du XIII ${ }^{\mathrm{e}}$ siècle», dans L'Argent au Moyen Âge..., p. 267-287; EAD., «Le vocabulaire de la vie économique dans les textes pastoraux des frères mendiants au XIII ${ }^{\mathrm{e}}$ siècle », dans L'economia dei conventi..., p. 151-186; G. ToDESCHINI, Ricchezza francescana..., ad indicem notamment à propos des sermons de Bernardin de Sienne.

54. P. WolfF, Commerce et marchands de Toulouse (vers 1350-vers 1450), Paris, 1954, p. 609-616; C. BEC, Les Marchands écrivains. Affaires et humanisme à Florence : 1375-1434, Paris et La Haye, 1967; ID., Les Livres des Florentins (1413-1608), Florence, 1984.

55. G. Todeschini, «Theological Roots of the Medieval/Modern Merchants'SelfRepresentation », dans M.-C. JАCOB, C. SECRETAn éd., The Self-Perception of early Modern Capitalists, New York, 2008, p. 17-46. 
étaient diffuses à la fin du Moyen Âge, circulaient, au moins dans certaines régions d'Europe et dans certains milieux.

Que le sens des mots change ou non dans le temps et l'espace - ce qui doit précisément être déterminé et mesuré puisqu'ils n'ont rien d'universel, d'objectif ou d'intemporel -, on ne peut ignorer, nous disent Sylvain Piron et Paolo Evangelisti, que les discours des théologiens de la fin du Moyen Âge sur les contrats, le crédit, l'argent, la monnaie, la valeur, l'utilité commune trouvèrent des prolongements, ne serait-ce qu'à travers l'emploi, tout d'abord chez les humanistes ${ }^{56}$, des mêmes mots ou des mêmes associations de mots dont certains furent forgés au Moyen Âge ${ }^{57}$. Cela, répétons-le, sans faire une histoire des filiations de doctrines ni rechercher les germes d'une pensée moderne. Plusieurs de ces notions prirent une importance centrale dans les réflexions de la seconde scolastique puis dans l'économie politique et la science économique des siècles suivants, où certains éléments des discussions médiévales furent même parfois repris par certains auteurs ${ }^{58}$. Paolo Evangelisti signale par exemple les points communs, à propos de l'impossibilité de changer la valeur de la monnaie pour accroître le patrimoine public, entre Eiximenis et Juan de Mariana (1536-1624), qui est reconnu comme une référence par John Milton et John Locke $^{59}$. Nombre de questions soulevées par Eiximenis sont aussi au cœur

56. $C f$. par exemple, pour un aperçu, H. BARON, «Franciscan Poverty and Civic Wealth as Factors in the Rise of Humanistic Thought», Speculum, 1 (1938), p. 1-37; P. GILl, «La place de l'argent dans la pensée humaniste au $\mathrm{XV}^{\mathrm{e}}$ siècle», dans L'Argent au Moyen Âge..., p. 309-326; G. Todeschini, Visibilmente crudeli..., p. 241 sq. ; Id., I mercanti..., p. 370 sq. ; C. Lenoble, «Leon Battista Alberti », dans P.-L. Porta, V. Zamagni éd., Il pensiero economico italiano, Rome, 2012, p. 73-80.

57. Sur ces mots, voir G. Todeschini, Il prezzo... et ID., I mercanti..., ad indicem; S. Piron, «Albert le Grand et le concept de valeur», dans R. LAMBERTInI, L. Sileo éd., I beni..., p. 131-156; ID., «L'apparition du resicum en Méditerranée occidentale, $\mathrm{XII}^{\mathrm{e}}-\mathrm{XIII}{ }^{\mathrm{e}}$ siècles », dans E. Collas-Heddeland, M. Coudry, O. Kammerer, A. J. Lemaître, B. Martin éd., Pour une histoire culturelle du risque. Genèse, évolution, actualité du concept dans les sociétés occidentales, Strasbourg, 2004, p. 59-76; G. Ceccarelli, «Le jeu comme contrat et le risicum chez Olivi», dans A. Boureau, S. Piron éd., Pierre de Jean Olivi (1248-1298). Pensée scolastique, dissidence spirituelle et société, Paris, 1999, p. 239-250; ID., «Risky Business: Theological and Canonical Thought on Insurance from the Thirteenth to the Seventeenth Century », Journal of Medieval and Early Modern Studies, 31/3 (2001), p. 607 658.

58. O. Langholm, «Olivi to Hutcheson: Tracing an Early Tradition in Value Theory », Journal of the History of Economic Thought, 31/2 (2009), p. 131-141 même si l'on se situe davantage dans 1'histoire des filiations de doctrine; S. PIron, Pierre de Jean Olivi..., p. 2425 .

59. P. Evangelisti, Il dodicesimo..., p. 74; ID., La bilancia della sovranità. Moneta, potere e cittadinanza in Europa (secc. XIV-XVIII), à paraître; on trouvera trace de l'influence du jésuite espagnol sur la science politique et économique anglaise, par exemple, chez J. E. E. Dalberg Acton, The History of Freedom and Other Essays, New York, 1993 (rééd. de textes datant de 1907-1908), p. 82; J. Neville FigGis, The Divine Right of Kings, Cambridge,

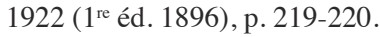


de la doctrine monétaire élaborée à partir de Jean Bodin et John Locke, mais Paolo Evangelisti mesure l'écart important qui sépare le franciscain catalan de ces auteurs ou des conceptions des fonctions de la monnaie chez Thomas Hobbes, notamment en raison du statut communautaire et républicain qu'Eiximenis accorde à la monnaie ${ }^{60}$.

Continuer de faire l'histoire sur la longue durée des mots, du lexique, de la construction des discours réemployés par l'économie politique moderne à partir du moment où celle-ci se définit elle-même comme telle, se pensa comme une science autonome et objet d'enseignement indépendant de la théologie morale, ne revient pas à croire que les écrits des théologiens des $\mathrm{XIII}^{\mathrm{e}}-\mathrm{XV}^{\mathrm{e}}$ siècles contiennent les idées encore à l'état brut et primitif de la pensée économique classique. Pour le dire avec Sylvain Piron, si le traité d'Olivi (comme celui d'Eiximenis) ne signe certainement pas la naissance de l'économie politique moderne, la scolastique fut néanmoins un laboratoire de certaines notions-clés de la pensée économique occidentale, ce qui justifie pleinement que l'on s'y intéresse en tant que tel, notamment en définissant les contextes particuliers dans lesquels ces notions furent forgées et le sens qu'elles assumèrent alors, comme on le fait désormais pour d'autres domaines de l'histoire des sciences ${ }^{61}$. Ou, pour citer Henri Bergson, «il faut des siècles de culture pour produire un utilitaire comme Stuart Mill ${ }^{62} »$.

L'histoire de ces continuités lexicales, pointées par Sylvain Piron et PaoloEvangelisti jusque chez les Lumières écossaises et la pensée républicaine anglaise du début $\mathrm{du} \mathrm{xx}^{\mathrm{e}}$ siècle, implique aussi de mesurer les écarts entre la pensée scolastique - au-delà même de sa dimension essentiellement caritative, spirituelle et salvifique - et la science politique moderne ou la science économique néoclassique. Dans cette perspective, la lecture en miroir des traités d'Olivi et d'Eiximenis (avec les analyses de leurs traducteurs) et du dernier ouvrage de l'économiste André Orléan sur la valeur nous semble revêtir une valeur heuristique non négligeable en permettant peut-être de mieux apprécier cette distance nécessaire à une meilleure compréhension de ces écrits du XIV ${ }^{\mathrm{e}}$ siècle ${ }^{63}$. André Orléan décrit l'économie comme un discours normatif qui dit ce qui est et ce qui doit être pour produire le réel, et l'on ne peut éviter de penser aux traités des scolastiques dont les fonctions et les objectifs pourraient être définis à peu près dans les mêmes termes. La critique de l'économie néoclassique par André Orléan dévoile - pour peu que l'on en compare les termes avec ce que nous savons désormais de la scolastique - ce qui pourrait être considéré

60. P. Evangelisti, Il dodicesimo..., p. 60-61, 74; Id., «Ad invicem... », § 66.

61. S. Piron, Pierre de Jean Olivi..., p. 12, 25.

62. H. Bergson, Les Deux Sources de la morale et de la religion, Paris, 2013 (1932), p. 126 .

63. A. ORlÉAn, L'Empire de la valeur. Refonder l'économie, Paris, 2011. 
en revanche comme l'une des différences fondamentales entre la science économique moderne et le discours des théologiens médiévaux. À la suite des analyses d'Émile Durkheim et de François Simiand, il montre que la théorie néoclassique met en œuvre des modèles abstraits reposant sur des paradigmes qui ne sont pas forcément fondés scientifiquement. Le rapport entre économie et pratiques réelles serait biaisé, selon lui et pour résumer très rapidement son livre, par les hypothèses de la maximisation de l'intérêt individuel et de la valeur-utilité qui fait de la valeur une grandeur objective préexistant aux échanges et s'imposant à la manière d'un fait naturel. Nous savons déjà que la pensée scolastique ne connaît pas ce principe et lui préfère une formation du «juste prix » dans l'échange, une évaluation au cas par cas fondée sur des critères variables, non objectifs et non absolus. Si l'on suit cette analyse de l'économie moderne et si cette théorie de la valeur est bien l'un des principes par lequel la science économique abstrait une catégorie particulière de pratiques du contexte et de l'ordre social dans lequel elles sont immergées ${ }^{64}$ - et rend de fait la réalité plus difficilement intelligible -, en l'ignorant, la pensée scolastique se différencierait de la doctrine néoclassique par un contact plus direct, pourrait-on dire, et peutêtre moins dissimulé, avec les réalités sociales de son temps.

Le langage «économique» des scolastiques fut produit moins comme le fruit d'une réflexion abstraite, théorique ou philosophique que dans un contexte historique concret et particulier, pour l'essentiel celui des problèmes liés à la gestion des biens d'Église et des activités marchandes et politiques des cités méditerranéennes entre $\mathrm{XII}^{\mathrm{e}}$ et $\mathrm{XIV}^{\mathrm{e}}$ siècles. Dans ce contexte, il constitua une œuvre multiple de formalisation de réalités mouvantes en pleine transformation. Loin d'être abstrait, ce langage est celui d'une pratique gouvernementale très concrète. Ce lexique toujours plus diversifié, technique et raffiné, en vint à traduire les réalités sociales et politiques en les fondant sur certaines formes historiquement datées d'échanges contractuels, d'évaluation et d'usages de la monnaie qui permettaient la justification et la reproduction d'un ordre oligarchique dont les marchands étaient les parangons et les personnes communes jugées inexpertes la contre-figure ${ }^{65}$. André Orléan estime que «l'évaluation n'a rien de neutre», «elle est l'acte par lequel la société s'engage en décidant quelles voies seront explorées et quelles autres rejetées ${ }^{66} »$. L'évaluation médiévale, on l'a vu, n'échappe pas à cette définition puisque ce sont bien des intérêts dominants qui en définissent les règles, les méthodes et les

64. P. Bourdieu, Les Structures sociales de l'économie, Paris, 2000, p. 11, définit en ces termes le processus d'abstraction «originaire» par lequel se constitue la science économique.

65. Cela, à partir du moment où ces formes contractuelles et la monnaie avaient été définies comme les instruments de rapports de fidélité aussi bien envers la communauté qu'envers Dieu.

66. A. ORLÉAN, L'Empire..., p. 139-141. 
critères, qui jugent des rapports d'équité et des équilibres, qui établissent la nature de ce qui est nécessaire ou superflu en fonction de la condition de chacun, et qui désignent non seulement ce qui, mais aussi qui, est utile et profitable à l'intérêt «commun » et au maintien de la justice. Penser que la théologie morale était peut-être plus proche des réalités sociales de son temps que ne l'est la science économique moderne n'implique donc aucune nostalgie. Comme l'a montré Giacomo Todeschini dans ses deux ouvrages les plus récents, ces discours élaborés à la fin du Moyen Âge relèvent de la formalisation et de la défense de rapports de domination appuyés sur un ordre mettant en jeu et valorisant la possession de dispositions techniques et de capitaux aussi bien matériels que symboliques ${ }^{67}$. Si et dans quelle mesure l'économie politique et la théorie néoclassique, l'économie de marché moderne, nos propres conceptions et catégories et les formes contemporaines de démocraties libérales ont hérité ou non de ces conceptions et de ce type de fonctionnements, d'autres que les médiévistes devraient être en mesure de nous l'apprendre.

Clément Lenoble - CNRS, CIHAM-UMR 5648

67. G. Todeschin, Visibilmente crudeli; ID., Come Giuda..., passim. 


\section{Langues d'Angleterre,}

coordonné par Alban Gautier et Jean-Pascal Pouzet

5 Alban Gautier et Jean-Pascal Pouzet

Les langues de

l'Angleterre médiévale : au-delà du bilinguisme

\section{David Trotter}

Peut-on parler de judéoanglo-normand?

Textes anglo-normands en écriture hébraíque

35 Christopher Lucken

Le beau français

d'Angleterre. Altérité de l'anglo-normand et invention du bon usage

57 Aude Mairey John Gower ou le multilinguisme en action

73 Catherine Nall et Daniel Wakelin Le déclin du multilinguisme dans The Boke of Noblesse et son Codicille de William Worcester
Essais et recherches

93 Donatella Nebbiai

Les livres de Jean

Durand († 1416),

"physicien »

et astrologue

\section{Lucie Laumonier}

En prévision des vieux

jours: les personnes âgées à Montpellier à la fin du Moyen Âge

\section{Points de vue}

147 François Foronda Procès politiques: une manie française?

161 Clément Lenoble Monnaie, valeur et citoyenneté chez Olivi et Eiximenis. "Moralisation de l'économie » ou "économie politique" médiévale?

181 Notes de lecture 205 Livres reçus 